Linguistique, littérature, didactique

\title{
Le figement des " actes de langage stéréotypés » en français et en allemand
}

\section{Maurice Kauffer}

\section{(2) OpenEdition}

\section{Journals}

Édition électronique

URL : http://journals.openedition.org/pratiques/2817

DOI : $10.4000 /$ pratiques. 2817

ISSN : 2425-2042

Éditeur

Centre de recherche sur les médiations (CREM)

Édition imprimée

Date de publication : 15 décembre 2013

Pagination : 42-54

Référence électronique

Maurice Kauffer, «Le figement des « actes de langage stéréotypés » en français et en allemand », Pratiques [En ligne], 159-160 | 2013, mis en ligne le 30 juin 2016, consulté le 30 avril 2019. URL : http:// journals.openedition.org/pratiques/2817 ; DOI : 10.4000/pratiques.2817

(c) Tous droits réservés 


\section{Le figement des « actes de langage stéréotypés » en français et en allemand}

\section{Maurice Kauffer}

Université de Lorraine-Nancy - CNRS-ATILF, UMR 7118

\section{Le figement : universalité et complexité}

Avant de définir ce que nous entendons par « actes de langage stéréotypés » français et allemands et de nous concentrer sur leur figement pragmatique, nous aimerions souligner au préalable certains aspects théoriques du figement qui nous seront utiles pour la suite de notre démonstration.

Le figement, longtemps négligé, voire ignoré en linguistique, connaît à présent un retour en grâce, pour plusieurs raisons (Mejri 2002 : 213), et est devenu l'objet de nombreuses études théoriques et empiriques. Il est même considéré comme une « donnée de base » (Mejri $2003: 24)$, voire un « phénomène central du langage » (Lamiroy 2008 : 89). Mejri ( ibid.) en détaille fort bien l'importance quantitative et la multidimensionnalité. Son importance s'est en effet avérée essentielle aussi bien dans le cadre d'une perspective lexicologique du figement pour le français que d'une perspective phraséologique pour l'allemand - et d'autres langues européennes - comme le montre Gréciano $(2003: 42)^{(1)}$. Il constitue encore pour la majorité des phraséologues de l'allemand un des critères fondamentaux de définition des phraséologismes, par exemple pour Gréciano (1997, entre autres) et Burger (2010 : 14), ce dernier détaillant bien la complexité du figement («Festigkeit») à trois niveaux (p. 15 et s.) : l'usage, la dimension psycholinguistique (globalité du phraséologisme) et les irrégularités structurelles. Gülich \& Krafft (1997) considèrent même que la notion de " préfabriqué » est le socle fondateur de la phraséologie. Ce caractère « universel » du figement est pointé d'une part par Mejri (2002 : 214), selon lequel « tout segment du discours est candidat au figement», d'autre part par Lamiroy (2008) pour qui toute « routinisation » d'une expression est susceptible d'en entraîner le figement. Même le renouvellement des possibilités de dénomination et de formation lexicale et la mise en place de la fonction expressive en dépendent (Mejri 2003 : 26 et 36, 2002 : 221 ; Lecolle 2006 : 32).

(1) Pour Gréciano (2003), le figement « s'étend et s'enracine » aussi dans les textes spécialisés, voire dans la terminologie. 
L'analyse du figement est cependant d'une rare complexité, due à plusieurs raisons. C'est en premier lieu le caractère multifactoriel des critères du figement (Lamiroy $2008: 89$ et $2010: 8$ ). Ces critères se situent à des niveaux très variés : lexical, phonétique, morphologique, syntaxique, sémantique (voir Mejri 2003 : 25 et s., Gross 1996 : 9 et s.), mais aussi cognitif et psychologique (Dubois 1997, Dobrovol'skij \& Piirainen 2009, Dobrovol'skij 2004 et sous presse). En outre, le figement a également une dimension pragmatique, encore peu explorée, et que nous examinerons plus loin. Le figement des expressions est donc apparemment une combinaison de différents figements à différents niveaux, selon une pondération de critères, voire une alchimie qu'il s'agit encore de quantifier. Remarquons par exemple avec Gross (1996:12) et Lamiroy (2010:18) que 1'opacité sémantique d'une expression est étroitement liée aux restrictions transformationnelles et aux irrégularités morphosyntaxiques auxquelles elle est soumise, mais ce lien doit encore être précisé.

Cette multifactorialité du figement a deux corollaires, qui renforcent encore sa complexité. D'une part son caractère scalaire, graduel, que nombre d'études ont souligné (entre autres Martin 1997, Gross 1996 : 16, Mejri 2002 : 215). En effet, les paramètres de figement comme (pour ne citer que les plus importants), la noncompositionnalité du sens — sur laquelle Perrin (sous presse 2013b : 2) s'interroge à juste titre —, les « anomalies » morpho-syntaxiques (Stein 2004), le « blocage des paradigmes synonymiques » (Gross 1996 : 17) etc., s'appliquent de manière très diversifiée aux expressions figées. Ces critères ne concernent que des sous-ensembles d'expressions dont l'extension varie selon chacun de ces critères, ils s'appliquent en outre avec une portée plus ou moins étendue à une expression donnée, et, pour compliquer les choses, ces expressions peuvent en outre avoir un degré de polylexicalité plus ou moins important. Ce caractère graduel est luimême à plusieurs dimensions car non seulement le figement global mais aussi la plupart des paramètres du figement sont graduels. Cela nous mène à une densification de la complexité que 1'on formule habituellement en termes imagés comme « treillis d'ordres partiels » (Achard \& Fiala 1997) ou « gamme infinie de possibilités » (Martin 1997). Les conséquences sont aussi de deux ordres. Soit le recours à une définition large du figement linguistique : Perrin (2013b:1) le définit comme « ce qui entrave la mobilité de toute combinatoire morpho-syntaxico-sémantique ou inférence pragmatique [...]», Anscombre \& Mejri (2011) le qualifient de «parole entravée » dans le titre de leur ouvrage. Soit une solution plus radicale, à savoir considérer la notion de figement comme «trop vague» (Mel'čuk 2011:50) et en revenir à une définition stricte, de nature statistique (cooccurrence des composantes de l'expression) et syntaxique.

\section{Phraséologismes pragmatiques et « actes de langage stéréotypés »}

\subsection{Phraséologismes pragmatiques}

Nous comptons nous pencher ici sur un type de figement que l'on peut appeler « figement pragmatique ». Il est plus particulièrement fréquent dans les « phraséologismes pragmatiques », c'est-à-dire ceux dont la fonction essentielle est non pas de nature référentielle ou dénotative ${ }^{(2)}$ mais se situe au niveau de la communica-

(2) C'est aussi une forme d' «aréférenciation» (terme employé par Mejri 2002: 217 pour le figement sémantique), due cette fois à la pragmaticalisation de l'expression (pragmaticalisation au sens de Dostie 2004). 
tion. Examinons donc d'abord en quoi consistent ces phraséologismes pragmatiques ${ }^{(3)}$. La plupart des publications récentes qui abordent ce domaine, d'ailleurs plutôt mieux labouré pour l'allemand que pour le français (Burger 2010, Hyvärinen 2011, Stein 1995 et 2004, Lüger 1999 et 2007, Dziadkiewicz 2007, Lamiroy 2010), proposent habituellement une typologie dont le critère de base est le lien fonctionnel existant ou non entre l'expression et la situation extra-linguistique. Beaucoup d'études (voir surtout Hyvärinen 2011, Burger 2010, Lüger 2007 et le précurseur Coulmas 1981) distinguent un premier type d'expressions, souvent appelées « Routineformeln » (formules routinières) qui sont étroitement liées à la situation : elles sont en général monofonctionnelles, et forment assez souvent, mais pas toujours, un énoncé autonome. Elles peuvent servir à exprimer un commentaire du locuteur, mais leur fonction essentielle est de réagir de façon stéréotypique dans des situations d'interaction sociale : formules de politesse, de contact etc., par exemple bon rétablissement!, sincères condoléances ! guten Tag (= bonjour). Une fonction complémentaire peut être également de nature sociale, par exemple le locuteur montre en employant la formule qu'il respecte les rites et usages sociaux (Lüger 2007 : 44). Le deuxième type est constitué par les expressions généralement indépendantes de la situation : les formules conversationnelles («Gesprächsformeln » pour Hyvärinen, ibid.). Elles ont des fonctions de communication variées dans le cadre de l'organisation des dialogues, mais aussi de la structuration des textes et de la mise en place des relations entre les partenaires de la communication (Burger 2010 : 56, Stein 1995 et 2004) et ne sont pas très souvent des énoncés autonomes. Par exemple à mon avis? ; tu vois? ; pas vrai?.

Au-delà des différences de détail entre les typologies, sur lesquelles nous ne nous attarderons pas ici, se posent deux problèmes pour ces phraséologismes pragmatiques. Premièrement, le fait que les critères définitoires des phraséologismes ( $c f$. note 3 ) ont tendance à ne pas toujours être respectés pour cette catégorie. Ainsi la polylexicalité n'est pas toujours de mise pour les phraséologismes pragmatiques, car certains sont monolexicaux : salut! ; Pustekuchen! (= pas du tout!) ; tintin!. De même, 1'idiomaticité sémantique, c'est-à-dire la non-compositionnalité, en principe constitutive d'un phraséologisme, peut être très faible ( $a$ mon avis), ou bien peut varier selon le contexte. Ainsi, tu vois ce que je vois? peut être employé soit au sens compositionnel, et donc être une question sur ce que les partenaires de la communication sont censés voir, soit pour marquer l'étonnement, l'incrédulité du locuteur. Autre exemple : (Na) warte mal! (=attends voir!) peut servir à solliciter une attente (verbe warten) ou bien à exprimer un avertissement, une menace ${ }^{(4)}$.

Deuxièmement, un critère nous paraît insuffisamment pris en compte, celui du statut d'énoncé des phraséologismes pragmatiques. Ces derniers sont-ils des énoncés, c'est-à-dire des actes de communication autonomes ${ }^{(5)}$ ou seulement des parties d'énoncé ? Certaines études font certes de ce statut d'énoncé un critère, mais elles n'aboutissent pas toujours à une catégorie précise de phraséologismes.

(3) Sont des phraséologismes, selon Burger (2010:14) — dont la définition est largement acceptée par la communauté des phraséologues de l'allemand - , des expressions qui remplissent trois critères : polylexicalité, figement et idiomaticité sémantique.

(4) Pour tenter de régler ce problème, Hyvärinen recourt à la notion (inspirée par la théorie du prototype) de «phraseologische Peripherie»; Stein, lui, (1995:42) propose de recourir à des critères de définition graduels.

(5) Pour définir un énoncé, nous retenons la notion d' « unité minimale communicative » («kommunikative Minimaleinheit ») de Zifonun, Hoffmann et Strecker (1997: 91). 
Par exemple, dans sa classification des phraséologismes, Burger (2010:38 et s.) définit ceux qui ont le statut de phrases («satzwertige Phraseologismen »), mais cette catégorie est hétérogène car très vaste : elle regroupe aussi bien les « feste Phrasen », c'est-à-dire pour Burger des phraséologismes ayant un lien avec le contexte, que les topos («topische Formeln»), à savoir les proverbes, lieux communs etc. ${ }^{(6)}$ Lüger (1999 et 1996) analyse aussi la catégorie des « satzwertige Phraseologismen », avec un résultat similaire. D'un autre côté, Hyvärinen (2011 : 40) considère comme acquis que le statut d'énoncé n'est pas constitutif d'une catégorie de phraséologismes, mais ne le démontre pas de façon convaincante. En revanche, l'analyse des « routines conversationnelles » ou «phrases figées » de Klein \& Lamiroy (2011) est tout à fait intéressante : nos deux collègues les définissent en effet comme des «phrases complètes (avec sujet grammatical figé) qui fonctionnent comme des énoncés automatiques [...]». Inversement, d'autres analyses, comme Bidaud (2002) et ses «structures figées de la conversation », Schneider (1989) et ses « expressions d'illocutoire stéréotypé » ou Schemann (1993) et ses « actes de langage lexicalisés » ne différencient pas clairement les phraséologismes pragmatiques ayant statut d'énoncé et les autres ${ }^{(7)}$.

\section{2. «Actes de langage stéréotypés » (= ALS)}

Il s'avère donc utile de définir et analyser une nouvelle catégorie d'expressions, que nous appellerons « actes de langage stéréotypés » (= ALS). Pour quelles raisons? D'une part, cette catégorie est à notre avis fonctionnellement cohérente et permet de comprendre la formation et l'emploi d'expressions fort courantes, surtout dans les dialogues, mais pas facilement analysables ${ }^{(8)}$ : une première recherche a permis d'inventorier près de 470 candidats ALS en français et environ autant en allemand. D'autre part, c'est une catégorie jusqu'à présent non encore analysée ni définie en tant que telle, sauf dans des études empiriques partielles ${ }^{(9)}$. Enfin, cette catégorie se prêtera bien de par sa définition à un examen du figement pragmatique, qui nous occupera ci-dessous. Ces ALS sont par ailleurs l'objet d'un dictionnaire actuellement en cours d'élaboration à l'Université de Lorraine (Nancy) par le GLFA (Groupe de lexicographie franco-allemande) que j'ai la tâche de coordonner ${ }^{(10)}$.

Les trois critères définitoires des ALS sont les suivants :

(6) S'y ajoutent les problèmes suivants : d'une part, Burger ne définit pas ce qu'il entend par «Phrase », d'autre part il reconnaît lui-même (ibid., p. 40) la difficulté de délimiter les « feste Phrasen » des formules routinières.

(7) Pour des précisions, voir Kauffer (2012a) et Gualberto-Schneider, Kauffer \& NahonRaimondez (sous presse).

(8) Fléchon, Frassi \& Polguère (2012:81) remarquent également, en introduisant les pragmatèmes, que «Les énoncés les plus courants et les plus anodins font partie de ceux dont il est le plus difficile de rendre compte en linguistique ».

(9) Les études les plus proches de notre analyse sont celles de Klein \& Lamiroy (2011) et Schemann (1993), avec les restrictions déjà énoncées plus haut.

(10) C'est un dictionnaire bilingue (français/allemand), contextuel c'est-à-dire comprenant de nombreux exemples des ALS en discours ainsi que de leurs traductions dans l'autre langue, et basé sur un corpus franco-allemand de bi-textes en grande partie constitué par les soins du GLFA. Pour des précisions sur ce projet, voir Métrich (2011), Gualberto-Schneider, Kauffer \& Nahon-Raimondez (sous presse), Kauffer (2012b et sous presse) et lire les premières microstructures publiées : Gualberto \& Balnat (2012), Kauffer (2012c), Métrich (2012), Nahon-Raimondez (2012), Pernot (2013), Keromnes (2013). 
* Primo, le statut d'énoncé. Un ALS peut, par définition, être un énoncé à part entière ${ }^{(11)}$. Ainsi, la belle affaire est bien un énoncé dans :

Il souffrira? La belle affaire! Tout le monde souffre. (Mauriac, p. 129).

C'est aussi le cas de na warte (= attends voir !) dans :

Na warte! Heute Nacht werde ich dich mit meinem Diktiergerät aufnehmen, du wirst schon sehen! (Buron, p. 15).

Voici d'autres exemples d'ALS qui sont bel et bien des énoncés : tu parles !; ben voyons! ; des clous! ; das ist die Höhe (= c'est le comble) ; denkste! (= tu parles !).

** Secundo, l'idiomaticité sémantique, à savoir la non-compositionnalité du sens de l'expression, sens qui n'est donc pas dérivable du sens de ses composantes. C'est par exemple le cas de $t u$ vas voir ce que tu vas voir !, bien différent d'une simple exclamation sur le fait de voir un objet, ou bien d'allons donc ! qui est loin d'une exhortation à se déplacer, ou encore de c'est le bouquet! où il ne s'agit guère de fleurs... En allemand, citons : das ist die Höhe!, qui signifie c'est le comble et non c'est la hauteur (au sens propre), denkste (= tu parles!), où il ne s'agit pas de «penser»(denken) etc.

*** Tertio, un ALS a une fonction essentiellement pragmatique, qui, pour simplifier, se situe beaucoup plus au niveau de la communication que de la référence. C'est une sorte d'acte de langage ${ }^{(12)}$ qui a une force illocutoire indéniable car il ne sert pas seulement à dire mais à réaliser quelque chose ${ }^{(13)}$. Au-delà de son contenu propositionnel, un ALS sert en général aussi à modifier la communication dans laquelle il est impliqué ou à exprimer une attitude ou un sentiment qui change d'une certaine façon les rapports existant entre le locuteur et son interlocuteur. L'ALS constitue habituellement une réaction à un procès ou à un énoncé mais il peut avoir aussi comme fonction de faire réagir l'interlocuteur. Voici des ALS exprimant ${ }^{(14)}$ :

- l'approbation ou un jugement positif : tu l'as dit (bouffi) ! ; c'est du billard! ; klarer Fall! ; du sagst es !,

— le refus : des clous! ; tu peux toujours courir! ; compte là-dessus! ; cause toujours (tu m'intéresses)! ; ach was! ; denkste!,

— l'étonnement: tu vois ce que je vois? ; première nouvelle! ; sieh mal (einer) an! ; au Backe! ; Mensch Meier!,

- la menace ou l'avertissement : essaie voir! ; tu vas voir (ce que tu vas voir)! ; mein lieber Freund!,

- la colère, l'indignation : c'est le bouquet! ; ça va pas la tête? ; das ist der Gipfel!,

— la volonté de tirer un bilan négatif : nous voilà bien! ; pas de bol!; Pech gehabt!,

— la résignation : c'est comme ça! ; les carottes sont cuites! ; was solls?

(11) Cela n’empêche certes pas que dans certains contextes il soit employé en tant que partie d'énoncé, mais même dans ce cas il peut être isolé en tant qu'énoncé. La belle affaire de garder les enfants ce week-end! est équivalent à : Garder les enfants ce week-end? La belle affaire!

(12) Nous n'approfondirons pas ici la notion d'actes de langage, for bien analysée par KerbratOrecchioni (2001), car cela dépasserait notre propos.

(13) Pour reprendre la célèbre formule d'Austin : « quand dire c'est faire ».

(14) Cette liste de fonctions pragmatiques n'est pas exhaustive, loin de là. Voir une liste plus complète dans Kauffer (2013 sous presse). 
Nous reviendrons dans la troisième partie sur la nature de cette fonction pragmatique des ALS.

\section{Le figement pragmatique des « actes de langage stéréotypés »}

Nous partons de l'hypothèse que le figement des ALS peut être de nature morpho-syntaxique, sémantique ou pragmatique. Cette hypothèse a déjà été en partie testée pour ce qui est des deux premières formes de figement, à savoir morphosyntaxique et sémantique. Nous nous y sommes consacré d'une part de façon synthétique dans Gualberto, Kauffer \& Nahon-Raimondez (sous presse) et Kauffer (2012a), d'autre part de manière plus précise dans les microstructures détaillées d'ALS déjà publiées dans le cadre du dictionnaire des ALS de notre équipe nancéienne ${ }^{(15)}$. Il résulte en gros deux choses de cette analyse. Le figement morphosyntaxique des ALS français et allemands examinés jusqu'à présent semble en général assez fort, en particulier pour les variations des catégories de temps, de personne et de nombre, le blocage des expansions syntaxiques et la limitation paradigmatique. Le figement sémantique est, lui, nettement plus variable car la noncompositionnalité du sens des ALS peut certes être forte, mais aussi faible dans un certain nombre de cas.

Peut-on à présent supposer qu'il existe un figement de nature pragmatique? Rey (1997 : 334) pense certes que « toute étude de la phraséologie conduit à intégrer les résultats de l'analyse syntagmatique et sémantique dans une perspective pragmatique » et, plus loin (ibid., p. 338), que « la phraséologie toute entière est affaire d'usage ». C'est là un jugement général, mais qui impliquerait qu'une dimension pragmatique du figement existerait forcément. Perrin (sous presse 2013 b : 2), est aussi d'avis que « [les formules énonciatives ${ }^{(16)}$ ] résultent d'un processus de figement diachronique aboutissant au codage linguistique de la valeur pragmatique indicielle [...]». Nous proposons de décrire le figement pragmatique d'un ALS sous la forme d'un ou plusieurs liens, plus ou moins étroits, existant entre d'une part l'ALS et de l'autre le contexte, la situation ou la fonction pragmatique de cet ALS. Si des liens étroits existent, ils « entraveraient » l'emploi de $1^{\prime} \operatorname{ALS}^{(17)}$ et constitueraient une forme de figement.

\subsection{Le lien de l'ALS avec le contexte ${ }^{(18)}$}

C'est sans doute le type de figement pragmatique le moins difficile à montrer. Nous ne citerons qu'un phénomène particulièrement intéressant pour les ALS. C'est le cas de ceux qui comprennent soit des pronoms anaphoriques (par exemple $c e, c ̧ a^{(19)}$, das) reprenant un élément du cotexte amont, soit des exophoriques dont

(15) Voir : Kauffer (2012c), Métrich (2012), Gualberto \& Balnat (2012), Nahon-Raimondez (2012), Pernot (2013), Keromnes (sous presse).

(16) Ces «formules énonciatives » ont en commun avec nos ALS une forme de grammaticalisation, voire de pragmaticalisation «par délexicalisation de l'expression » (ibid.).

(17) $C f$. les remarques de Perrin (2013b:1) et Anscombre \& Mejri (2011) sur le figement comme « entrave ", dans la première partie de cet article.

(18) Nous entendons ici contexte au sens de contexte linguistique et discursif (ou cotexte) mais aussi (pour les exophoriques suivants) le contexte externe, à savoir les éléments extra-linguistiques, situationnels. Voir la distinction des différents types de contexte dans le bel article de Kerbrat-Orecchioni (2012:13).

(19) Klein \& Lamiroy (2011 : 204) s'interrogent d'ailleurs à juste titre sur la valeur du pronom ce/ça qui est parfois le seul sujet possible. 
le sens ne peut être correctement interprété que par rapport à la situation. Ces derniers peuvent être de nature temporelle (jetzt, maintenant), spatiale (ici, hier, da) ou être des pronoms personnels et des possessifs de la 1e et 2e personne. On les trouve dans des ALS comme:

ça la fout mal ; mon ail! ; comme tu y vas! ; tu me la copieras ; les bras m'en tombent ; vate faire voir!,

das ist die Höhe! (= c'est le comble); nun/jetzt wird's aber Tag! (= c'est incroyable !) ; jetzt ist aber Sense (= ça suffit maintenant) ; hat man da noch Worte? (= c'est inouï); da lachen ja die Hühner! (= c'est complètement ridicule).

Klein \& Lamiroy $(2011: 200)$ remarquent aussi le grand nombre de « sujets pronominaux exprimant la deixis dans les phrases figées ». Observons cependant que les pronoms de la 1e et 2e personne des ALS perdent en grande partie leur valeur exophorique quand ils sont figés. Par exemple le pronom $t u$ est figé dans tu parles! ${ }^{(20)}$ car on ne peut envisager un autre pronom personnel comme sujet de cet ALS. Mais tu n'est pas figé dans tu peux (toujours) te l'accrocher! ou tu en as de bonnes! car d'autres personnes sont envisageables : il peut (toujours) se l'accrocher! ; elle en a de bonnes! (21)

\subsection{Le lien de l'ALS avec la situation extra-linguistique}

Il s'agit tout d'abord du lien avec la situation qui est nécessaire pour décrypter les exophoriques présents dans les ALS : nous l'avons abordé dans la partie précédente et n'y reviendrons donc pas.

D'autre part, on ne peut pas dire qu'il existe pour les ALS un lien étroit avec la situation. C'est plutôt le cas des « Routineformeln», que nous avons définies dans notre deuxième partie, qui sont quasi systématiquement appelées par une situation d'interaction précise. Par exemple herzliches Beileid (= sincères condoléances) ne sera en principe prononcé qu'à l'intention d'une personne endeuillée, gute Besserung! (= bon rétablissement) seulement si l'interlocuteur est malade et en voie de guérison. Il s'agit ici de ce que Fonagy (1997 : 131) appelait déjà des « énoncés liés », à savoir " presque automatiquement déclenchés par telle ou telle situation caractéristique ». Mel'čuk (2011:44) définit également les « pragmatèmes » comme des phrasèmes « contraints » par rapport à une situation extralinguistique ${ }^{(22)}$.

Pour les ALS, en revanche, ce lien avec la situation est beaucoup moins fort ${ }^{(23)}$. On le remarque en observant deux phénomènes. D'une part, une situation particulière n'est pas forcément susceptible de déclencher un seul ALS mais peut appeler plusieurs ALS très différents : tout dépend en fait de la fonction pragmatique de l'ALS, de sa valeur illocutoire dans la communication. Par exemple, si l'on reprend la situation ci-dessus, à savoir un locuteur qui apprend que son interlocuteur est malade, on peut tout à fait imaginer que le locuteur réagisse en employant un

(20) Un seul cas, relativement marginal, de vous parlez ! a été trouvé dans notre corpus (Métrich $2012: 3$ ).

(21) Rappelons que dans sa classification des phraséologismes, Burger (2010:39) crée à l'intérieur de ceux à statut de phrase une sous-catégorie, les «feste Phrasen », qui ont comme caractéristique d'être explicitement rattachés au contexte grâce à un élément déictique, anaphorique ou autre.

(22) Gréciano (2003: 48) parle pour ces expressions de «figement externe».

(23) Même si, comme le fait observer Mel'čuk (2011:49), toute unité linguistique peut être contrainte par la situation. 
des ALS suivants : Les bras m'en tombent! exprimerait sa stupéfaction, il ne manquait plus que ça! sa colère ; que veux-tu! sa résignation et je te vois venir son incrédulité face à cette information... D'autre part, un même ALS peut être employé dans plusieurs situations bien différentes. Et dans de nombreux cas, c'est même la situation qui permettra de donner à une certaine expression sa valeur d'ALS ou bien non. Par exemple, c'est le bouquet! peut être la réponse à la question c'est le bouquet ou la bouteille que tu apportes? qui vise à savoir quel cadeau sera apporté lors d'une invitation à un dîner. Dans ce cas, c'est le bouquet! n'est pas un ALS. Mais lorsque cette même expression sert dans une autre situation à exprimer son indignation à propos d'un acte jugé comme répréhensible, c'est le bouquet! aura le sens de c'est le comble!, cela va trop loin! et ce sera un ALS avec une valeur illocutoire de refus et d'indignation. De même, on peut imaginer que je te vois venir! en tant que non-ALS soit prononcé en réaction à une situation où l'interlocuteur se dirige vers le locuteur tout en essayant de se cacher : le locuteur exprime qu'il voit effectivement son interlocuteur se diriger vers lui malgré les efforts de ce dernier de se dissimuler aux regards du locuteur. En revanche, je te vois venir! est un ALS dans une situation où le locuteur veut manifester une certaine incrédulité critique à l'égard du comportement de son partenaire.

Remarquons en définitive deux choses. D'abord il n'y a pas de «routinisation» d'un ALS dans une situation particulière, c'est-à-dire un emploi systématique d'un ALS dans une certaine situation. D'autre part, on peut même affirmer que le lien entre ALS et situation s'avère ténu et très variable. Il faut donc bel et bien comprendre contexte au sens de « situation » lorsque Hyvärinen (2011:11) parle d'une réalisation contextuelle de la fonction de certains phraséologismes ${ }^{(24)}$ et également lorsque Klein \& Lamiroy (2011 : 195) déclarent que les «phrases figées » - fort proches de nos ALS —, « ne prennent leur sens qu'à l'intérieur du contexte ${ }^{(25)}$ dans lequel elles sont énoncées » ${ }^{(26)}$. Remarquons enfin que la notion de situation est souvent, il faut le dire, en grande partie intuitive et qu'il convient de lui conférer plus de rigueur, ce que tente d'ailleurs Bladas (2012) pour les formules de routine, en appliquant la notion de subjectivation.

\subsection{Le lien de l'ALS avec la fonction pragmatique}

La «fonction pragmatique » de l'ALS est le troisième critère définitoire des ALS que nous avons examiné dans notre deuxième partie. Cette fonction pragmatique est l'intention communicative exprimée par le locuteur grâce à l'ALS, c'està-dire le fait que par 1'ALS les données de la communication entre les interlocuteurs sont modifiées. Y a-t-il un lien étroit entre ALS et fonction pragmatique, qui aboutirait donc à une sorte de figement pragmatique ? Quelles seraient les différentes dimensions de ce lien?

En fait, un même ALS peut avoir plusieurs fonctions pragmatiques, qui peuvent être très différentes, voire opposées ${ }^{(27)}$. Métrich (2012) a bien montré dans la microstructure de $t u$ parles! que cet ALS peut exprimer soit la contestation dans l'exemple suivant :

(24) A savoir : «kontextbedingte Realisierung des Funktionspotenzials von Phraseologismen ».

(25) C'est moi qui met en italiques.

(26) Voir aussi Lamiroy $(2010: 110)$ qui constate que l'interprétation de certaines expressions est fonction de la situation d'énonciation.

(27) Hyvärinen (2011:41) parle également de polyfonctionnalité («Multifunktionalität») des phraséologismes pragmatiques, mais sans donner de précisions. 
- [...] J'étais une vraie petite sainte Thérèse de Lisieux.

— Tu parles! Une démone, oui. (N. de Buron, p. 69)

soit l'approbation, ce qui une fonction pour le moins très différente :

- T'as eu les jetons?

— Tu parles. Jamais eu une telle trouille de ma vie. Même pendant les bombardements. (R. Queneau, p. 49).

Quelquefois les choses sont moins tranchées et ces fonctions pragmatiques différentes sont des variantes fonctionnelles ayant un point commun pragmatique de nature plus globale. J'ai déjà montré par exemple ${ }^{(28)}$ que 1'ALS la belle affaire sert de façon générale à minimiser plus ou moins fortement un énoncé ou un procès, ce qui peut donner les variantes suivantes. Soit le locuteur veut banaliser, voire approuver une affirmation ou un procès :

- Est-ce que tu peux garder mon mouflet pendant les vacances?

- La belle affaire! (Schneider, p. 126),

Soit il réduit une affirmation à néant, la frappe en quelque sorte de «non-pertinence » :

- Il a trouvé ton carnet, et il l'a lu en cachette. [...]

- La belle affaire. Il n'a rien pu comprendre. (Troyat, p. 64),

Soit il exprime un refus, voire un défi :

Ils cherchent à obtenir ma main, la belle affaire! Je suis riche et mon père avancera son gendre (Stendhal, p. 330).

Un dernier point est le fait qu'il existe un certain nombre d'ALS ayant des fonctions pragmatiques très voisines, mais se différenciant néanmoins de plusieurs façons possibles. Par exemple, il en existe beaucoup qui expriment le refus :

allons donc! ; de l'air! ; des clous! ; du balai ! ; du vent! ; tu peux toujours courir! ; tu veux rire? ; compte là-dessus! ; cause toujours (tu m'intéresses)!

ach was! ; ach wo! ; denkste! ; Pustekuchen ; rutsch mir den Buckel runter! ; steig mir den Buckel rauf!

On s'aperçoit à la lecture de ces ALS que le refus peut être plus ou moins ferme, ou bien il peut être proche de l'incrédulité, ou bien il peut être accompagné de l'expression de sentiments complémentaires comme la colère ou l'indignation, ou bien il peut être verbalisé dans des registres de langue différents ${ }^{(29)}$ etc., bref il y a un grand nombre de variantes pragmatiques. Il s'agit là d'un domaine qui demande encore à être exploré de façon plus précise, à l'aide d'exemples authentiques basés sur un corpus suffisamment important pour constituer un thésaurus des emplois possibles ${ }^{(30)}$.

Après avoir rappelé certains points théoriques importants du figement, en particulier les raisons qui fondent son universalité et sa complexité, nous avons centré

(28) Voir Kauffer (2012a).

(29) Rappelons qu'une liste indicative d'ALS français et allemands classés par fonction pragmatique se trouve dans Kauffer (2013, sous presse).

(30) C'est pourquoi nous travaillons dans le cadre de notre projet lexicographique avec un corpus électronique de bi-textes français et allemands de plusieurs centaines d'ouvrages numérisés. 
notre analyse sur le figement des « actes de langage stéréotypés » en français et en allemand, Il s'agit d'expressions couramment employées mais encore peu étudiées et que nous avons définies par rapport aux phraséologismes pragmatiques et aux formules de routine. Nous avons exploré leur figement morpho-syntaxique, en général assez fort, leur figement sémantique, très variable, mais surtout les aspects pragmatiques de leur figement, en particulier l'existence de liens plus ou moins étroits entre ces expressions et leur contexte, leur situation et leur fonction pragmatique. Ces résultats, ainsi que les perspectives de recherche se dessinant après cette analyse, permettent à notre avis de faire avancer la recherche sur le figement linguistique et sur la phraséologie du français et de l'allemand.

\section{Bibliographie}

ACHARD, P. \& FIALA, P. (1997), «La locutionalité à géométrie variable», in: Fiala, P., Lafon, P. \& Piguet, M.-F. (éds.), La locution : entre lexique, syntaxe et pragmatique. Paris, Klincksieck, 273-284.

ANDERSEN, H. L. (2007), « Marqueurs discursifs propositionnels », Langue française 154, 13-28.

Anscombre, J.-Cl. \& MeJRI, S. (éd.) (2011), La parole entravée : études sur le figement. Paris, Champion.

BIDAUD, F. (2002), Structures figées de la conversation. Analyse contrastive françaisitalien. Bern, Lang.

BlADAS, O. (2012), "Conversational routines, formulaic language and subjectification », Journal of pragmatics 44, 929-957.

Burger, H. (2010), Phraseologie. Eine Einführung am Beispiel des Deutschen, 4. Auflage. Berlin, Schmidt.

Coulmas, F. (1981), Routine im Gespräch. Zur pragmatischen Fundierung der Idiomatik. Wiesbaden, Athenaion.

DoBROVOL'SKIJ, D. (sous presse), «Image component of idiom semantics : its nature and functions », in: Gautier, L. (éd.). Perspectives franco-allemandes sur le figement, coll. Kontraste/Contrastes. Studien zum deutsch-französischen Sprach- und Diskursvergleich 3. Frankfurt/Main, etc., Peter Lang.

- (2004), "Idiome aus kognitiver Sicht », in : Steyer, K. (ed.), Wortverbindungen - mehr und weniger fest, Institut für Deutsche Sprache, Jahrbuch 2003. Berlin \& New York, De Gruyter, 117-131.

Dobrovol'skiJ, D. \& Pitrainen, E. (2009), Zur Theorie der Phraseologie Kognitive und kulturelle Aspekte. Tübingen, Stauffenburg.

Dostie, G. (2004), Pragmaticalisation et marqueurs discursifs. Analyse sémantique et traitement lexicographique. Bruxelles, de Boeck Duculot.

Dubois, D. (1997), "Catégories, prototypes et figements », in : Martins-Baltar, M. (ed.), La locution entre langue et usages. Paris, ENS Editions/Ophrys, 103-129.

DZIADKIEWICZ, A. (2007), « La traduction automatique de phraséologismes pragmatiques : quelles représentations à travers la diversité formelle et structurelle ? ", Corela 5(2), http ://edel.univ-poitiers.fr/corela/ document.php ?id=1825. (30 décembre 2007.) 
Fléchon, G., Frassi, P. \& POlguère, A. (2012), « Les pragmatèmes ont-ils un charme indéfinissable ? », in : Ligas, P. \& Frassi, P., Lexiques. Identités, Cultures. Vérone, QuiEdit, 81-104.

FÓNAGY, I. (1997), «Figement et changements sémantiques », in : Martins-Baltar, Michel (ed.), La locution entre langue et usage. Paris, ENS Editions/Ophrys, 131-164.

GrÉCIANO, G. (2003), « Le figement s'étend et s'enracine », Cahiers de lexicologie 1, 41-49.

— (1995), «Fachphraseologie », in : Métrich, René / Faucher, Eugène (eds.), Rand und Band - Abgrenzung und Verknüpfung als Grundtendenzen des Deutschen. Tübingen, Narr, 183-195.

Gross, G. (1996), Les expressions figées en français. Gap \& Paris, Ophrys.

GuAlBerto-SCHNEIDER, A., BALnAt, V. (2012), « Petit dictionnaire permanent des "actes de langages stéréotypés" (ALS) - Microstructure de "Na warte !" ", Nouveaux Cahiers d'allemand 3, 269-284.

GÜLICH, E. \& KRAFFT, U. (1997), « Le rôle du "préfabriqué" dans les processus de production discursive », in : Martins-Baltar, M. (ed.), La locution entre langue et usages. Paris, ENS Editions/Ophrys, 241-276.

HYVÄRINEN, Irma (2011), "Zur Abgrenzung und Typologie pragmatischer Phraseologismen », in : Hyvärinen, I. \& Liimatainen, A. (ed.) : Beiträge zur pragmatischen Phraseologie, Finnische Beiträge zur Germanistik 25. Frankfurt a. M., Peter Lang, 9-43.

KAUfFER, M. (2013, sous presse), «Phraseologismen und stereotype Sprechakte im Deutschen und im Französischen », Linguistik on line, 18 p.

- (2012a), «Plaidoyer pour une phraséologie pragmatique sur la base des ALS français et allemands ", in: Ortiz A., M. L. (ed.) : Tendências atuais na pesquisa descritiva e aplicada em fraseologia e paremiologia (Current trends in descriptive and applied research on phraseology and paremiology), Anais, Volume 2. Campinas SP, Pontes Editores, 192-208.

- (2012b), «Petit dictionnaire permanent des "actes de langages stéréotypés" (ALS) - Introduction », Nouveaux Cahiers d'allemand 1, 1-3.

- (2012c), «Petit dictionnaire permanent des "actes de langages stéréotypés" (ALS) - Microstructure de "Das ist die Höhe !" ". Nouveaux Cahiers d'allemand 2, 129-145.

- (2011), «Actes de langage stéréotypés en allemand et en français. Pour une redéfinition du stéréotype grâce à la phraséologie ", Nouveaux Cahiers d'allemand 1, 35-53.

Kerbrat-OrecCHIONI, C. (2012), « Le contexte revisité », in : CORELA $-n^{\circ}$ thématique RJC Cotexte, contexte, situation (http ://corela.edel.univpoitiers.fr/index.php ?id=2627), $28 \mathrm{p}$.

- (2001), Les actes de langage dans le discours. Paris, Nathan.

Keromnes, Y. (2013), "Petit dictionnaire permanent des "actes de langages stéréotypés" (ALS) - Microstructure de "Et puis quoi encore !" ", Nouveaux Cahiers d'allemand 2, 141-149.

KLEIN, J.-R. \& LAMIROY, B. (2011), « Routines conversationnelles et figement », in: Anscombre, J.-Cl. \& Mejri, S. (éd.) : La parole entravée : études sur le figement, Paris, Champion, 195-213.

LAMiroy, B. (2010), Les expressions figées de la francophonie. Paris, Ophrys. - (2008), "Les expressions figées : à la recherche d'une définition », in : Blumenthal, P. \& Mejri, S., Les séquences figées : entre langue et discours (ZfSL-Beiheft 36). Stuttgart, Steiner, 85-98. 
LeColle, M. (2006), "Changement dans le lexique - changement du lexique : lexicalisation, figement, catachrèse ", in : Lecolle, M. \& Leroy, S. (ed)., Changements linguistiques : figement, lexicalisation, grammaticalisation (Cahiers de praxématique 46). Montpellier, Presses Universitaires de la Méditerrannée, 23-42.

LÜGER, H.-H. (2007), « Pragmatische Phraseme : Routineformeln », in : Burger, H., Dobrovol'skij, D., Kühn, P. \& Norrick, N. R. (ed.), Phraseologie / Phraseology Ein internationales Handbuch zeitgenössischer Forschung 1, 444-459. Berlin \& New York, Mouton/De Gruyter.

- (1999), Satzwertige Phraseologismen. Eine pragmalinguistische Untersuchung. Wien, Praesens.

- (1996), "Satzwertige Phraseologismen im Text - Elemente eines Mehrebenenmodells. Beiträge zur Fremdsprachenvermittlung 30, 76-103.

Martin, R. (1997), "Sur les facteurs du figement lexical », in : Martins-Baltar, M. (ed.), La locution entre langue et usages. Paris, ENS Editions/Ophrys, 291-305.

MEJRI, S. (2003), « Le figement lexical », Cahiers de lexicologie 82, 23-39.

- (2002), «Le figement lexical : nouvelles tendances », Cahiers de lexicologie 80, 213-223.

MEL'ČUK, I. (2011), «Phrasèmes dans le dictionnaire », in: Anscombre, Jean-Claude \& Mejri, Salah. (éd.) (2011), La parole entravée : études sur le figement, Paris, Champion, 41-61.

MÉTRICH, R. (2012), "Petit dictionnaire permanent des “actes de langages stéréotypés" (ALS) - Microstructure de "tu parles !" ". Nouveaux Cahiers d'allemand 1, 3-17.

- (2011), "Zur Konzeption eines bilingualen Wörterbuchs der stereotypen Sprechakte am Beispiel von tu parles! », in: Schäfer, P. \& Schowalter, C. (ed.), In mediam linguam. Mediensprache - Redewendungen - Sprachvermittlung. Festschrift für Heinz-Helmut Lüger. Landau, Verlag Empirische Pädagogik, 331-348.

NAHON-RAimondeZ, A.-M. (2012), « Petit dictionnaire permanent des "actes de langages stéréotypés" (ALS) - Microstructure de "N'empêche !" ". Nouveaux Cahiers d'allemand 4, 367-381.

PERNOT, C. (2013), «Petit dictionnaire permanent des “actes de langages stéréotypés” (ALS) - Microstructure de "Et comment !" ». Nouveaux Cahiers d'allemand 1, $1-12$.

PERRIN, L. (sous presse 2013a), "Les formules monologiques et dialogiques de l'énonciation », in : Ducart, D., Dufaye, L. \& Gournay, L., Les théories énonciatives aujourd'hui : un demi-siècle après Benveniste. Paris, Ophrys, 187211.

— (sous presse 2013b), « Du figement au défigement des unités lexicales », Cahiers de lexicologie 2.

REY, A. (1997), "Phraséologie et pragmatique », in : Martins-Baltar, M. (éd.), La locution entre langue et usages, Paris, ENS éditions, 333-347.

Schemann, H. (1993). Deutsche Idiomatik - Die deutschen Redewendungen im Kontext. Stuttgart \& Dresden, Klett.

SCHNEIDER, F. (1989), Comment décrire les actes de langage? - De la linguistique pragmatique à la lexicographie : "La belle affaire » et «Tu m'en diras tant! ». Tübingen, Niemeyer.

STEIN, S. (2004), «Formelhaftigkeit und Routinen in mündlicher Kommunikation », in: Steyer, Kathrin (ed.), Wortverbindungen - mehr und weniger fest, Institut für Deutsche Sprache, Jahrbuch 2003. Berlin \& New-York, De Gruyter, 262-288. 
- (1995), Formelhafte Sprache. Untersuchungen zu ihren pragmatischen und kognitiven Funktionen im gegenwärtigen Deutsch. Frankfurt/Main, Lang.

Zifonun, G., HofFMAnN, L. \& STRECKER, B. (1997), Grammatik der deutschen Sprache, 3 vol. Berlin/New York, De Gruyter.

\section{Sources}

BURON, N. de (1999), Liebling, hörst du mir zu ?, traduction de R. Walther. Berlin, Ullstein.

- (1998), Chéri, tu m'écoutes?. Paris, Plon.

Mauriac, F. (1938), Asmodée. Paris, Grasset.

Queneau, R. (1967), Zazie dans le métro. Paris, Gallimard.

Stendhal (1983), Le Rouge et le Noir. Paris, Librairie Générale Française.

TROYAT, H. (1947), Tant que la terre durera. Paris, La Table Ronde.

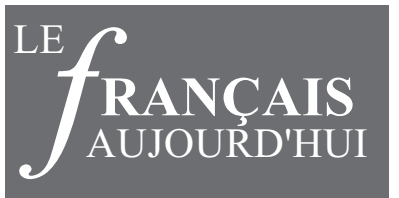

\section{HISTOIRE DES ARTS : DE LA NOTION A LA DISCIPLINE}

Numéro coordonné par J.-M. Baldner et A. Barbaza

Histoire des arts : de la notion à la discipline - J.-M Baldner, A. Barbaza

Arts plastiques et Histoire des arts, identités et territoires disciplinaires C. Herth

Constellations et racontages pour des histoires des arts comme expériences -

S. Martin

L'Histoire des arts et le temps à l'œuvre - S. Fabre

De la lecture de l'image à l'enseignement de l'Histoire des arts - G. Di Rosa

Histoire des arts et jeux de langage : comment parler de l'œuvre ? -

J.-C. Chabanne

"Giotto, c'est vieux et pas très bien dessiné ! "- O. Sidokpohou

Écrire pour devenir un spectateur amateur- C. Archat-Tatah

Quel Art ? Quelle École ? - C. Ruby

Revue de l'Association Française des Enseignants de Français http://www.afef.org

Courriel editeur : infos@armand-colin.fr

Courriel rédaction : le-francais-aujourdhui@wanadoo.fr

N 182 - septembre 2013 - ISBN 978-2-200-92848-3 